\title{
Chemical abundances in 43 metal-poor stars
}

\author{
Bengt Gustafsson ${ }^{1}$, Martin Asplund ${ }^{2}$, Bengt Edvardsson ${ }^{1}$, Karin \\ Jonsell $^{1}$, Pierre Magain ${ }^{3}$, and Poul Erik Nissen ${ }^{4}$ \\ ${ }^{1}$ Department of Astronomy and Space Physics, Box 515, SE751 20 Uppsala, Sweden \\ email:first name.family name@astro.uu.se \\ ${ }^{2}$ Mt Stromlo Observatory, Australian National University \\ email:martin@mso.anu.edu.au \\ ${ }^{3}$ Institute of Astrophysics and Geophysics, Université de Liège, Belgium \\ email: pierre.magain@ulg.ac.be \\ ${ }^{4}$ Institute of Physics and Astronomy, Århus University, Denmark \\ email: pen@phys.au.dk
}

\begin{abstract}
We have derived abundances of $\mathrm{O}, \mathrm{Na}, \mathrm{Mg}, \mathrm{Al}, \mathrm{Si}, \mathrm{Ca}, \mathrm{Sc}, \mathrm{Ti}, \mathrm{V}, \mathrm{Cr}, \mathrm{Fe}, \mathrm{Ni}$ and $\mathrm{Ba}$ for 43 metal-poor field stars, mostly stars at the turn-off point and on the subgiant branch, in the interval $-3.0<[\mathrm{Fe} / \mathrm{H}]<-0.4$. The analysis is differential relative to the Sun. Oxygen abundances, with consideration of NLTE effects, were derived from the OI $777.4 \mathrm{~nm}$ triplet lines. We find $[\mathrm{O} / \mathrm{Fe}]$ to gradually increase with decreasing $[\mathrm{Fe} / \mathrm{H}]$, though considerably slower than has earlier been obtained from $\mathrm{OH}$ lines in the $\mathrm{UV}$. A scatter in $[\mathrm{O}, \mathrm{Mg}, \mathrm{Ca}, \mathrm{Ti} / \mathrm{Fe}]$ at a given $[\mathrm{Fe} / \mathrm{H}]$ is found and we argue that this scatter is partly real. The deviations from the mean trends of abundance ratios vs $[\mathrm{Fe} / \mathrm{H}]$ are found to correlate in non-trivial ways for different abundances. Similar trends are found from results of accurate studies by other groups. This seems to give further evidence for the hypothesis that the stars once formed in different subsystems, with different star-formation rates.
\end{abstract}

The paper is in press in A\&A, and may be obtained as astro-ph/0505118.

Keywords. Stars: abundances, Galaxy: halo, Galaxy: evolution. 


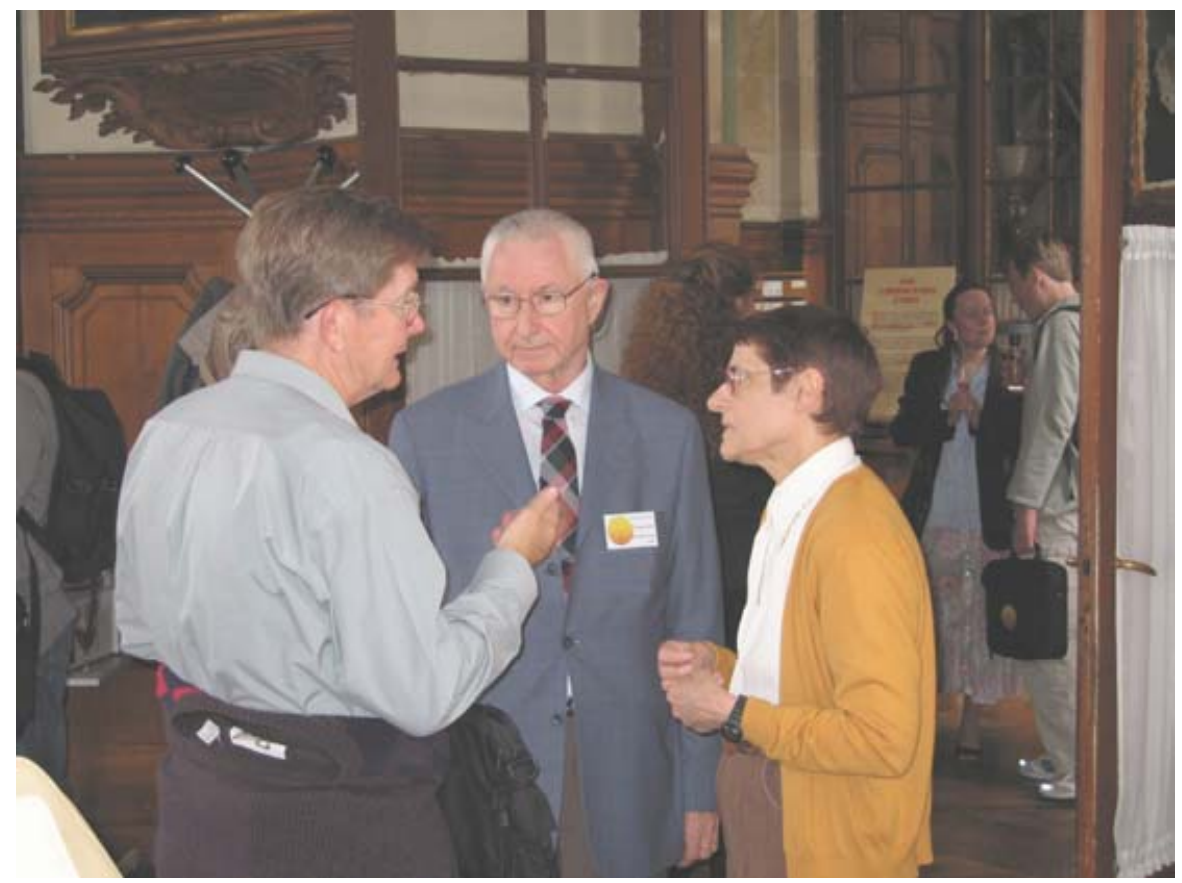

Monique and François Spite talking with John Norris at the welcome reception, in the Paris Observatory historical building.

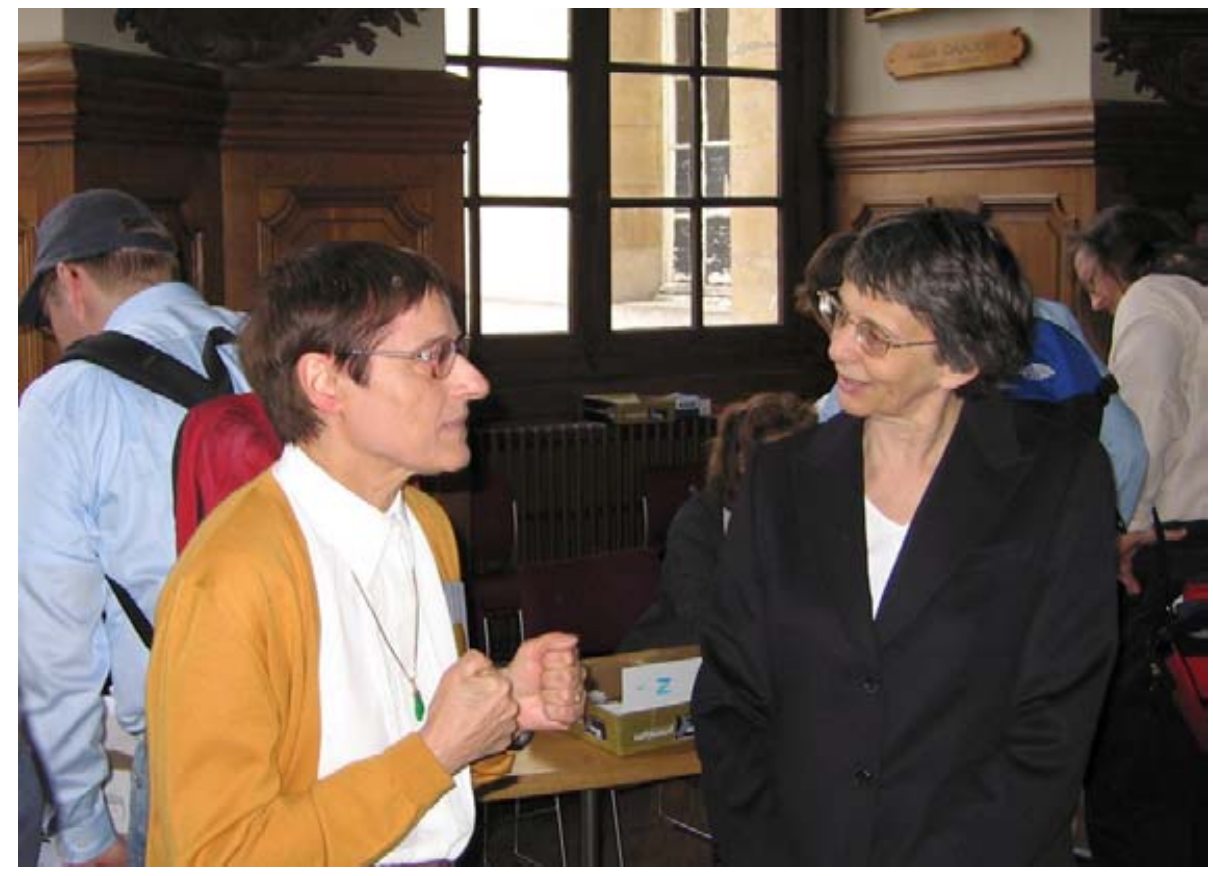

Monique Spite and Ruth Peterson at the welcome reception. 